\title{
SEVEN YEARS OF CRITICAL INCIDENTS IN CRITICAL CARE UNITS: DATA FROM THE NATIONAL BASE.
}

Palencia M., Galve A.I., Cabrerizo P., Portas M., Lema M., Fernández-Quero L. Gregorio Marañón University Hospital, Madrid, Spain.

\section{BACKGROUND}

$>$ Critical Care Units constitute areas especially vulnerable to the emergence of critical incidents $(\mathrm{Cl})$. In its genesis contribute multiple factors such as the patient pathology, communication barriers, the realization of a large number of activities per patient and day, the practice of diagnostic procedures and invasive treatments and the amount and complexity of information received. According to the various series described in the literature, the likelihood of an $\mathrm{Cl}$ is increased between $8 \%$ and $26 \%$ per day of stay, and the vast majority of incidents are preventable.

$>$ The goal of this study is to perform a review of all the $\mathrm{Cl}$ occurred in units of resuscitation and declared in SENSAR Database. SENSAR is a Spanish national critical incident reporting system in Anesthesia and Critical Units.

\section{MATERIALS AND METHODS}

We design a prospective observational study in which we analized the medication critical incidents reported by SENSAR during the period of time between January 2009 and October 2016. Review of all Cl reported in SENSAR January 2009 until November 2016

\section{RESULTS AND DISCUSSION}

$>$ In this period a total of $6205 \mathrm{Cl}$ were declared , 155 of the incidents had occurred in a Critical Care Unit, which constitutes $2,4 \%$ of the total.

$>51(32.9 \%)$ of them correspond to incidents of medication, 38 (24.5\%) are clinicians, $19(12.2 \%)$ to communication errors and $17(10.9 \%)$ to equipment.

$>$ With regard to morbidity we found that 91 (58.7\%) no caused harm, $23(14.8 \%)$ were less morbidity, 24 (15.4\%) intermediate morbidity and $14(9 \%)$ morbidity were greater. There were two (1\%) cases of death with contribution of the incident. There was no death declared directly related to the incident
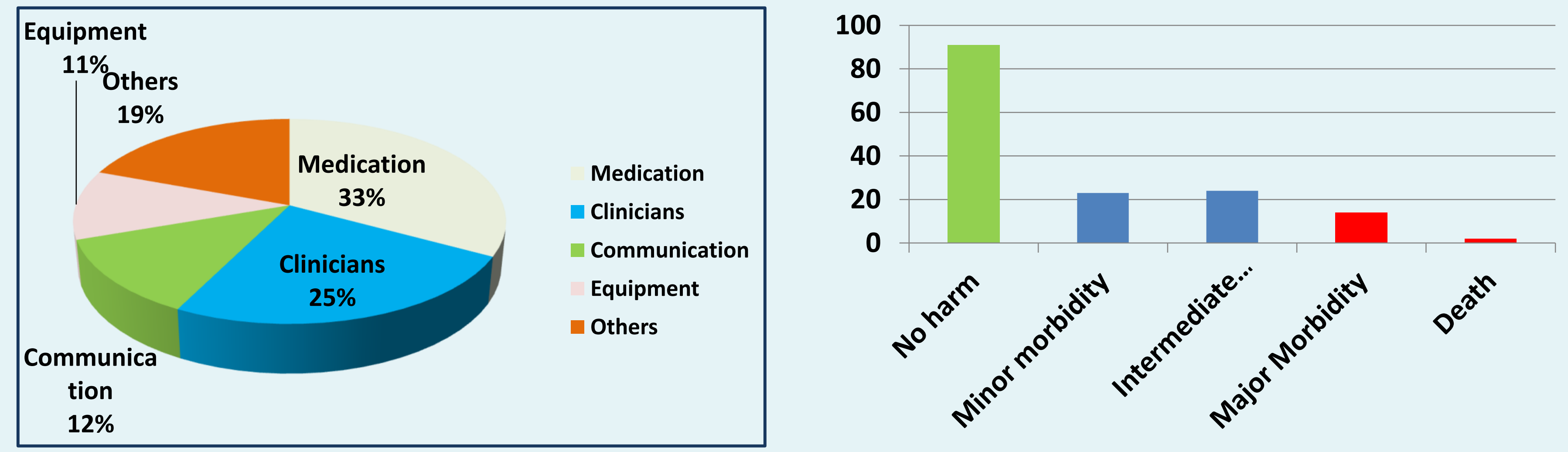

\section{CONCLUSIONS}

$>$ Emphasizes the low rate of declaration of $\mathrm{Cl}$ in Critical Care Units.

$>$ Due to the characteristics of the online SENSAR database it is not possible to find out how many of the hospitals have registered with these units among its facilities, the number of beds available, the type of patients that attend or the days of average stay, which undoubtedly affects the statistics.

$>$ Despite its limitations, has proved to be a tool that works and that is in progressive expansion, it is imperative to implement measures to enhance its use and the expansion of the modern culture of security in this environment are especially vulnerable, such as protocols for handover, safety courses multidisciplinary, simulation, sessions of service, talks of learning (debriefing) or training in crisis resolution and non-technical skills. 